\title{
The Imperative for Patient-Centred Research to Develop Better Quality Services in Rare Diseases
}

\author{
Karen Facey $\cdot$ Helle Ploug Hansen
}

Published online: 24 January 2015

(C) Springer International Publishing Switzerland 2015

\section{The Policy Imperative to Consider Rare Diseases Differently}

The definition of a rare disease differs country by country, from an occurrence of $<1$ per 10,000 people in Taiwan and China to $\leq 5$ per 10,000 people in Europe and Argentina. The issue everyone agrees with is that there are many, at least over 5,000 and perhaps even 6,000-8,000 different rare diseases. For many of these diseases, the prevalence is much lower than the defined threshold of rarity and they are genetic chronic diseases that impact daily life and quality of life, are life threatening and there is no approved disease-modifying or curative treatment. All of this means that patients can feel isolated and healthcare services often struggle to define and deliver good quality care, support and rehabilitation.

Efforts have been made to improve this situation with special regulatory incentives and assistance for the development of treatments for rare diseases. The US Food and Drug Administration was the first to establish a special process for medicines for rare diseases and, in 2000, the European Medicines Agency established its orphan medicinal products process. However, despite this, in the decade from 2000, only 108 products for rare conditions

\section{K. Facey $(\square)$}

Evidence Based Health Policy Consultant, Woodlands Lodge, Buchanan Castle Estate, Drymen G63 0HX, UK

e-mail: k.facey@btinternet.com

\section{H. P. Hansen}

Institute of Public Health, Research Unit: General Practice, University of Southern Denmark, J.B. Winsløws Vej 9,

5000 Odense C., Denmark

e-mail: HPHansen@health.sdu.dk were submitted to the European regulator and only 63 were authorised for treatment [1].

In 2009, the Council of the European Union recommended that Member States should take action on rare diseases and establish and implement strategies for rare diseases to ensure that patients with rare diseases have access to high-quality care including diagnostics, treatments and habilitation. The goal was to have these strategies in place by the end of 2013 and there has been a flurry of activity to see this achieved in Europe. Some countries have undertaken surveys to understand the issues faced by patients with rare diseases who are seeking to access services, and the following common themes arise: delay in diagnosis, lack of awareness of diseases in medical community, need to improve equity of access to services and effective treatments, fragmented non-specialist care and lack of research.

In this special edition of The Patient, we seek to demonstrate that valuable informative research can be undertaken in rare diseases that can inform all these issues. A range of studies show how qualitative and humanistic research can be used to understand the most challenging aspects of living with a rare disease, patients' unmet needs, patients attitudes to new treatments and how patients can influence the complex world of reimbursement. We have studies from researchers, clinicians and patients. They demonstrate how through collaboration they can learn from one another about research, living with an illness and decision making.

\section{Living with a Rare Disease}

Jeppesen et al. [2] show how with just six patients and intensive research over a year-long period, narrative 
journalistic stories can provide important new insights into the challenges of daily living with amyotrophic lateral sclerosis. Patients described the continuous process of creating a new normality of everyday life as the disease progressed. The narrative journalistic stories also revealed conflicting views between patients and professionals about the disease and prognosis, but also that the stories could help health professionals better understand the vulnerability and coping mechanisms of patients, their families and caregivers.

A mixed-methods approach was used by Gowran et al. [3] to understand the service needs of patients with epidermolysis bullosa (EB). Interviews and a workshop were held with key stakeholders, followed by a national survey of EB patient group members in Ireland. This research was constructed using the sustainable community of practice model focusing on place, people, pace and policy. It was undertaken in partnership with the patient group and included clear visuals such as word clouds and rich pictures. This resulted in an understanding of the complexities of life with $\mathrm{EB}$, from the challenges in meeting the primary needs of caring for those with $\mathrm{EB}$, through to service access issues and the complex emotional burden faced by caregivers.

\section{Patient Communities}

Doyle [4] explores the issues faced by children with cystinosis growing into adulthood, and their parents. Interviews and focus groups were used to understand the impact of the rare disease community and found that participants were comfortable in the disease community, which allowed them to share experiences, and to gain confidence to go public about their illness and mentor others.

The European Organization for Rare Diseases (EURORDIS) and the National Organization for Rare Diseases (NORD) in the USA are well established as effective networks for the representation of issues for patients with rare diseases, influencing policy and practice. However, other regions are less well served. For example, in the AsiaPacific region, rare disease patient organisations have been slower to form, but as Wong-Rieger et al. [5] state, recent workshops are exploring the development of an alliance of patient organisations in the region, learning from best practices of NORD and EURORDIS and engaging with all relevant stakeholders including policy makers.

\section{Attitudes to New Medicines}

In three focus groups, Kesselheim et al. [6] explored the views of patients, caregivers and patient representatives about new medicines. They discussed issues of paucity of scientific data and the need to balance risks and benefits. Bridges et al. [7] pick up this theme in their paper about patients with idiopathic pulmonary fibrosis. Analysis of interviews with patients, researchers and policy/industry informants showed that patients seemed to understand issues relating to emerging treatments. The documentation of patient experience (including burden of illness) and the challenge of coping with disease progression (to lung transplantation and end-of-life considerations) were key issues.

In response to the Food and Drug Administration's initiative to support patient-focused drug development, Parent Project Muscular Dystrophy collaborated with Hollin and colleagues to understand the treatment preferences of patients with Duchenne Muscular Dystrophy [8]. They tested the use of best-worse scaling vs conjoint analysis for 18 potential treatments with six attributes and three levels. One hundred and nineteen caregivers completed the survey and good concordance was found between methods for benefit-risk attributes but some differences for implications of nausea and knowledge about the medicine.

\section{Access to Medicines}

Access to medicines is often an important issue for those with rare diseases as the cost per patient can be extremely high because of the challenges of development. As Dragojlovic et al. [9] indicate, such treatments often do not meet traditional cost-effectiveness criteria, but not providing medically necessary care could be considered as being contrary to the principle of equity. Some countries have recently sought to identify whether society will pay extra for treatments for rare diseases but Dragojlovic et al. show that measurement of societal value is compromised by a low level of public awareness. Following a survey of over 2,000 citizens, they conclude that current estimates of societal value for treating rare diseases (via opportunity cost arguments) may not reflect citizens preferences and are not sufficiently robust to be incorporated into policy frameworks for reimbursement decisions about medicines for rare diseases.

Smit [10] provides us with telling personal reflections about his role as a patient representative in an appraisal committee, making decisions about two expensive medicines for very rare diseases. He clearly describes the difficult dilemmas faced when such decisions have to be made. However, he stresses that these challenges would be easier if patient groups were involved at every stage of decision-making processes for the assessment and reimbursement of medicines. This issue was explored by 
Menon et al. [11] in their systematic review, which found numerous opportunities for patients with rare diseases to get involved in the development and reimbursement of a health technology. In particular, they proposed greater involvement in the design and conduct of clinical trials and the valuation of evidence at the reimbursement stage. Menon et al. [11] used that systematic review at several conferences and workshops with patients and families from rare disease communities to discuss their best opportunities for involvement across the life cycle of a medicine. A policy framework was established [12] that identified the areas where uncertainties could be reduced by patient input in relation to evaluation of clinical benefit, value for money, affordability and adoption/diffusion of a medicine for a rare disease.

Smit [10] suggests that there is a gap between the evidence considered by health technology assessment and the issues that patients and their families consider to be important. To close that gap we need to generate robust high-quality research about patients' perspectives, experiences and preferences in a targeted way so that uncertainties can be reduced and the quality of care can be improved. As we have shown in this edition, this is not just achievable in rare diseases, in fact, patient-centred research provides a richness of perspectives and insights into living with a rare disease that can and should inform decisionmaking to improve the quality of care.

Acknowledgments Karen Facey has contracts with a range of health technology companies and undertakes paid and voluntary work for HTAi, patient organisations, and government agencies and committees.

\section{References}

1. Facey K, Granados A, Guyatt G, et al. Generating health technology assessment evidence for rare diseases. Int $\mathbf{J}$ Tech Ass Health Care. 2014. doi:10.1017/S02664623140000464.
2. Jeppesen J, Rahbek J, Gredal O, Hansen HP. How narrative journalistic stories can communicate the individual's challenges of daily living with amyotrophic lateral sclerosis. Patient. 2015. doi:10.1007/s40271-014-0088-6.

3. Gowran RJ, Kennan A, Marshall S, et al. Adopting a sustainable community of practice model when developing a service to support patients with epidermolysis bullosa (EB): a stakeholdercentered approach. Patient. 2015. doi:10.1007/s40271-014-00975.

4. Doyle M. Peer support and mentorship in a US rare disease community: findings from the cystinosis in emerging adulthood study. Patient. 2015. doi:10.1007/s40271-014-0085-9.

5. Wong-Rieger D, Claxton W, Vines R, et al. An Asia Pacific alliance for rare diseases. Patient. 2015. doi:10.1007/s40271-0140103-y.

6. Kesselheim AS, McGraw S, Thompson L, et al. Development and use of new therapeutics for rare diseases: views from patients, caregivers and advocates. Patient. 2015. doi:10.1007/s40271-0140096-6.

7. Bridges JFP, Paly VF, Barker E, Kervitsky D. Identifying the benefits and risks of emerging treatments for idiopathic pulmonary fibrosis: a qualitative study. Patient. 2015. doi:10.1007/ s40271-014-0081-0.

8. Hollin IL, Peay HL, Bridges JFP. Caregiver preferences for emerging Duchennemuscular dystrophy treatments: a comparison of best-worst-scaling and conjoint analysis. Patient. 2015. doi:10. 1007/s40271-014-0104-x.

9. Dragojlovic N, Rizzardo S, Bansback N, et al. Challenges in measuring the societal value of orphan drugs: insights from a Canadian stated preference survey. Patient. 2015. doi:10.1007/ s40271-014-00xx-x.

10. Smit C. Personal reflections of a patient representative in an appraisal committee. Patient. 2015. doi:10.1007/s40271-0140086-8.

11. Menon D, Stafinski T, Dunn A, Short H. Involving patients in reducing decision uncertainties around orphan and ultra-orphan drugs: a rare opportunity? Patient. 2015. doi:10.1007/s40271014-0106-8.

12. Menon D, Stafinski T, Dunn A, Wong-Rieger D. Developing a patient directed policy framework for managing orphan and ultraorphan drugs throughout their lifecycle. Patient. 2015. doi:10. 1007/s40271-014-00xx-x. 